\title{
FINDING OR NOT FINDING
} RULES IN TIME SERIES

\author{
Jessica Lin and Eamonn Keogh
}

\begin{abstract}
Given the recent explosion of interest in streaming data and online algorithms, clustering of time series subsequences has received much attention. In this work we make a surprising claim. Clustering of time series subsequences is completely meaningless. More concretely, clusters extracted from these time series are forced to obey a certain constraint that is pathologically unlikely to be satisfied by any dataset, and because of this, the clusters extracted by any clustering algorithm are essentially random. While this constraint can be intuitively demonstrated with a simple illustration and is simple to prove, it has never appeared in the literature. We can justify calling our claim surprising, since it invalidates the contribution of dozens of previously published papers. We will justify our claim with a theorem, illustrative examples, and a comprehensive set of experiments on reimplementations of previous work.
\end{abstract}

In econometrics, a large fraction of research has been devoted to time series analysis (Enders, 2003). As a recent trend, time series data have also been given a lot of attention in the data mining community (Keogh \& Kasetty, 2002; Roddick \& Spiliopoulou, 2002). This is highly anticipated since time series data has extended

\footnotetext{
Applications of Artificial Intelligence in Finance and Economics Advances in Econometrics, Volume 19, 175-201 Copyright $\odot 2004$ by Elsevier Ltd. All rights of reproduction in any form reserved ISSN: 0731-9053/doi:10.1016/S0731-9053(04)19007-5
} 
1 its influence outside of economic applications. It is a by-product in virtually every 2 human endeavor, including biology (Bar-Joseph et al., 2002), finance (Fu et al., 3 2001; Gavrilov et al., 2000; Mantegna, 1999), geology (Harms et al., 2002b), space 4 exploration (Honda et al., 2002; Yairi et al., 2001), robotics (Oates, 1999) and human motion analysis (Uehara \& Shimada, 2002). While traditional time series analysis focuses on modeling and forecasting, data mining researchers focus on discovering patterns (known or unknown) or underlying relationships among the data. These techniques can be very useful in aiding the decision-making process for the econometrics community.

Of all the techniques applied to time series, clustering is perhaps the most frequently used (Halkidi et al., 2001), being useful in its own right as an exploratory technique, and as a subroutine in more complex data mining algorithms (Bar-Joseph et al., 2002; Bradley \& Fayyad, 1998). The work in this area can be broadly classified into two categories:

- Whole Clustering: The notion of clustering here is similar to that of conventional clustering of discrete objects. Given a set of individual time series data, the objective is to group similar time series into the same cluster.

- Subsequence Clustering: Given a single time series, sometimes in the form of streaming time series, individual time series (subsequences) are extracted with a sliding window. Clustering is then performed on the extracted time series subsequences.

Subsequence clustering is commonly used as a subroutine in many other algorithms, including rule discovery (Das et al., 1998; Fu et al., 2001; Uehara \& Shimada, 2002; Yairi et al., 2001) indexing (Li et al., 1998; Radhakrishnan et al., 2000), classification (Cotofrei, 2002; Cotofrei \& Stoffel, 2002), prediction (Schittenkopf et al., 2000; Tino et al., 2000), and anomaly detection (Yairi et al., 2001). For clarity, we will refer to this type of clustering as STS (Subsequence Time Series) clustering.

In this work we make a surprising claim. Clustering of time series subsequences is meaningless! In particular, clusters extracted from these time series are forced to obey a certain constraints that are pathologically unlikely to be satisfied by any dataset, and because of this, the clusters extracted by any clustering algorithm are essentially random.

Since we use the word "meaningless" many times in this paper, we will take the time to define this term. All useful algorithms (with the sole exception of random number generators) produce output that depends on the input. For example, a decision tree learner will yield very different outputs on, say, a credit worthiness domain, a drug classification domain, and a music domain. We call an algorithm "meaningless" if the output is independent of the input. As we prove in this 
1 paper, the output of STS clustering does not depend on input, and is therefore 2 meaningless.

Our claim is surprising since it calls into question the contributions of dozens of papers. In fact, the existence of so much work based on STS clustering offers an obvious counter argument to our claim. It could be argued: "Since many papers have been published which use time series subsequence clustering as a subroutine, and these papers produced successful results, time series subsequence clustering must be a meaningful operation."

We strongly feel that this is not the case. We believe that in all such cases the results are consistent with what one would expect from random cluster centers. We recognize that this is a strong assertion, so we will demonstrate our claim by reimplementing the most successful (i.e. the most referenced) examples of such work, and showing with exhaustive experiments that these contributions inherit the property of meaningless results from the STS clustering subroutine.

The rest of this paper is organized as follows. In Section 2 we will review the necessary background material on time series and clustering, then briefly review the body of research that uses STS clustering. In Section 3 we will show that STS clustering is meaningless with a series of simple intuitive experiments; then in Section 4 we will explain why STS clustering cannot produce useful results. In Section 5 we show that the many algorithms that use STS clustering as a subroutine produce results indistinguishable from random clusters. We conclude in Section 6.

\section{BACKGROUND MATERIAL}

In order to frame our contribution in the proper context we begin with a review of the necessary background material.

\subsection{Notation and Definitions}

We begin with a definition of our data type of interest, time series:

Definition 1 (Time Series). A time series $T=t_{1}, \ldots, t_{m}$ is an ordered set of $m$ real-valued variables.

Data mining researchers are typically not interested in any of the global properties of a time series; rather, researchers confine their interest to subsections of the time series, called subsequences. 


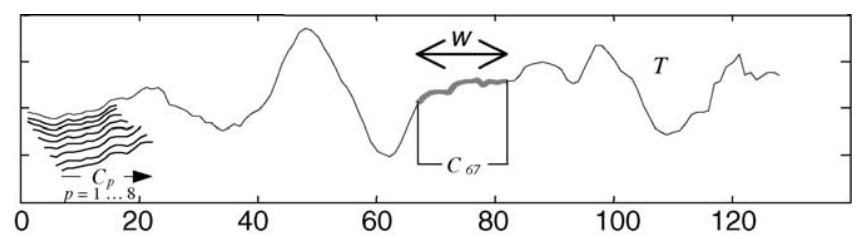

Fig. 1. An Illustration of the Notation Introduced in This Section: A Time Series T of Length 128, a Subsequence of Length $w=16$, Beginning at Datapoint 67, and the First 8 Subsequences Extracted by a Sliding Window.

Definition 2 (Subsequence). Given a time series $T$ of length $m$, a subsequence $C_{p}$ of $T$ is a sampling of length $w<m$ of contiguous positions from $T$, that is, $C=t_{p}, \ldots, t_{p+w-1}$ for $1 \leq p \leq m-w+1$.

In this work we are interested in the case where all the subsequences are extracted, and then clustered. This is achieved by use of a sliding window.

Definition 3 (Sliding Windows). Given a time series $T$ of length $m$, and a userdefined subsequence length of $w$, a matrix $S$ of all possible subsequences can be built by "sliding a window" across $T$ and placing subsequence $C_{p}$ in the $p$ th row of $S$. The size of matrix $S$ is $(m-w+1)$ by $w$.

Figure 1 summarizes all the above definitions and notations.

Note that while $S$ contains exactly the same information ${ }^{1}$ as $T$, it requires significantly more storage space.

\subsection{Background on Clustering}

One of the most widely used clustering approaches is hierarchical clustering, due to the great visualization power it offers (Keogh \& Kasetty, 2002; Mantegna, 1999). Hierarchical clustering produces a nested hierarchy of similar groups of objects, according to a pairwise distance matrix of the objects. One of the advantages of this method is its generality, since the user does not need to provide any parameters such as the number of clusters. However, its application is limited to only small datasets, due to its quadratic computational complexity. Table 1 outlines the basic hierarchical clustering algorithm.

A faster method to perform clustering is $k$-means (Bradley \& Fayyad, 1998). The basic intuition behind $k$-means (and a more general class of clustering algorithms known as iterative refinement algorithms) is shown in Table 2. 
Table 1. An Outline of Hierarchical Clustering.

The $k$-means algorithm for $N$ objects has a complexity of $\mathrm{O}(k N r D)$, where $k$ is the number of clusters specified by the user, $r$ is the number of iterations until convergence, and $D$ is the dimensionality of time series (in the case of STS clustering, $D$ is the length of the sliding window, $w$ ). While the algorithm is perhaps the most commonly used clustering algorithm in the literature, it does have several shortcomings, including the fact that the number of clusters must be specified in advance (Bradley \& Fayyad, 1998; Halkidi et al., 2001).

It is well understood that some types of high dimensional clustering may be meaningless. As noted by (Agrawal et al., 1993; Bradley \& Fayyad, 1998), in high dimensions the very concept of nearest neighbor has little meaning, because the ratio of the distance to the nearest neighbor over the distance to the average neighbor rapidly approaches one as the dimensionality increases. However, time series, while often having high dimensionality, typically have a low intrinsic dimensionality (Keogh et al., 2001), and can therefore be meaningful candidates for clustering.

\subsection{Background on Time Series Data Mining}

The last decade has seen an extraordinary interest in mining time series data, with at least one thousand papers on the subject (Keogh \& Kasetty, 2002).

Table 2. An Outline of the $k$-Means Algorithm.

Algorithm: $k$-means

1. Decide on a value for $k$.

2. Initialize the $k$ cluster centers (randomly, if necessary).

3. Decide the class memberships of the $N$ objects by assigning them to the nearest cluster center.

4. Re-estimate the $k$ cluster centers, by assuming the memberships found above are correct.

5. If none of the $N$ objects changed membership in the last iteration, exit. Otherwise goto 3. 
Tasks addressed by the researchers include segmentation, indexing, clustering, classification, anomaly detection, rule discovery, and summarization.

Of the above, a significant fraction use subsequence time series clustering as a subroutine. Below we enumerate some representative examples.

- There has been much work on finding association rules in time series (Das et al., 1998; Fu et al., 2001; Harms et al., 2002a; Uehara \& Shimada, 2002; Yairi et al., 2001). Virtually all work is based on the classic paper of Das et al. that uses STS clustering to convert real-valued time series into symbolic values, which can then be manipulated by classic rule finding algorithms (Das et al., 1998).

- The problem of anomaly detection in time series has been generalized to include the detection of surprising or interesting patterns (which are not necessarily anomalies). There are many approaches to this problem, including several based on STS clustering (Yairi et al., 2001).

- Indexing of time series is an important problem that has attracted the attention of dozens of researchers. Several of the proposed techniques make use of STS clustering (Li et al., 1998; Radhakrishnan et al., 2000).

- Several techniques for classifying time series make use of STS clustering to preprocess the data before passing to a standard classification technique such as a decision tree (Cotofrei, 2002; Cotofrei \& Stoffel, 2002).

- Clustering of streaming time series has also been proposed as a knowledge discovery tool in its own right. Researchers have suggested various techniques to speed up the STS clustering (Fu et al., 2001).

The above is just a small fraction of the work in the area, more extensive surveys may be found in (Keogh, 2002a; Roddick \& Spiliopoulou, 2002). MEANINGLESSNESS OF STS CLUSTERING

In this section we will demonstrate the meaninglessness of STS clustering. In order to demonstrate that this meaninglessness is a result of the way the data is obtained by sliding windows, and not some quirk of the clustering algorithm, we will also do whole clustering as a control (Gavrilov et al., 2000; Oates, 1999). We will begin by using the well-known $k$-means algorithm, since it accounts for the lion's share of all clustering in the time series data mining literature. In addition, the $k$-means algorithm uses Euclidean distance as its underlying metric, and again the Euclidean distance accounts for the vast majority of all published work in this area (Cotofrei, 2002; Cotofrei \& Stoffel, 2002; Das et al., 1998; Fu et al., 2001; 
1 Keogh et al., 2001), and as empirically demonstrate in (Keogh \& Kasetty, 2002)

2 it performs better than the dozens of other recently suggested time series distance 3 measures.

\subsection{K-means Clustering}

Because $k$-means is a heuristic, hill-climbing algorithm, the cluster centers found may not be optimal (Halkidi et al., 2001). That is, the algorithm is guaranteed to converge on a local, but not necessarily global optimum. The choices of the initial centers affect the quality of results. One technique to mitigate this problem is to do multiple restarts, and choose the best set of clusters (Bradley \& Fayyad, 1998). An obvious question to ask is how much variability in the shapes of cluster centers we get between multiple runs. We can measure this variability with the following equation:

- Let $A=\left(\bar{a}_{1}, \bar{a}_{2}, \ldots, \bar{a}_{k}\right)$ be the cluster centers derived from one run of $k$-means.

- Let $B=\left(\bar{b}_{1}, \bar{b}_{2}, \ldots, \bar{b}_{k}\right)$ be the cluster centers derived from a different run of $k$-means.

- Let $\operatorname{dist}\left(\bar{a}_{i}, \bar{a}_{j}\right)$ be the distance between two cluster centers, measured with Euclidean distance.

Then the distance between two sets of clusters can be defined as:

$$
\text { cluster_distance }(A, B) \equiv \sum_{i=1}^{k} \min \left[\operatorname{dist}\left(\bar{a}_{i}, \bar{b}_{j}\right)\right], \quad 1 \leq j \leq k
$$

The simple intuition behind the equation is that each individual cluster center in $A$ should map on to its closest counterpart in $B$, and the sum of all such distances tells us how similar two sets of clusters are.

An important observation is that we can use this measure not only to compare two sets of clusters derived for the same dataset, but also two sets of clusters which have been derived from different data sources. Given this fact, we propose a simple experiment.

We performed 3 random restarts of $k$-means on a stock market dataset, and saved the 3 resulting sets of cluster centers into set $\hat{X}$. We also performed 3 random restarts on random walk dataset, saving the 3 resulting sets of cluster centers into set $\hat{Y}$. Note that the choice of "3" was an arbitrary decision for ease of exposition; larger values do not change the substance of what follows.

We then measured the average cluster distance (as defined in Eq. 1), between each set of cluster centers in $\hat{X}$, to each other set of cluster centers in $\hat{X}$. We call 
this number within_set_ $\hat{X}_{-}$distance.

$$
\text { within_set_ } \hat{X}_{-} \text {distance }=\frac{\sum_{i=1}^{3} \sum_{j=1}^{3} \text { cluster_distance }\left(\hat{X}_{i}, \hat{X}_{j}\right)}{9}
$$

We also measured the average cluster distance between each set of cluster centers in $\hat{X}$, to cluster centers in $\hat{Y}$; we call this number beween_set_$\hat{X}_{-}$and__ $\hat{Y}_{-}$distance.

$$
\text { beween_set_} \_\hat{X}_{-} \text {and } \_\hat{Y}_{-} \text {distance }=\frac{\sum_{i=1}^{3} \sum_{j=1}^{3} \operatorname{cluster} \_ \text {distance }\left(\hat{X}_{i}, \hat{X}_{j}\right)}{9}
$$

We can use these two numbers to create a fraction:

$$
\text { clustering meaning fulness }(\hat{X}, \hat{Y}) \equiv \frac{\text { within_set_} \hat{X}_{-} \text {distance }}{\text { beween_set_} \hat{X} \_ \text {and_} \hat{Y} \_ \text {distance }}
$$

We can justify calling this number "clustering meaningfulness" since it clearly measures just that. If, for any dataset, the clustering algorithm finds similar clusters each time regardless of the different initial seeds, the numerator should be close to zero. In contrast, there is no reason why the clusters from two completely different, unrelated datasets should be similar. Therefore, we should expect the denominator to be relatively large. So overall we should expect that the value of clustering meaningfulness $(\hat{X}, \hat{Y})$ be close to zero when $\hat{X}$ and $\hat{Y}$ are sets of cluster centers derived from different datasets.

As a control, we performed the exact same experiment, on the same data, but using subsequences that were randomly extracted, rather than extracted by a sliding window. We call this whole clustering.

Since it might be argued that any results obtained were the consequence of a particular combination of $k$ and $w$, we tried the cross product of $k=\{3,5,7,11\}$ and $w=\{8,16,32\}$. For every combination of parameters we repeated the entire process 100 times, and averaged the results. Figure 2 shows the results.

The results are astonishing. The cluster centers found by STS clustering on any particular run of $k$-means on stock market dataset are not significantly more similar to each other than they are to cluster centers taken from random walk data! In other words, if we were asked to perform clustering on a particular stock market dataset, we could reuse an old clustering obtained from random walk data, and no one could tell the difference!

We re-emphasize here that the difference in the results for STS clustering and whole clustering in this experiment (and all experiments in this work) are due exclusively to the feature extraction step. In particular, both are being tested on the same datasets, with the same parameters of $w$ and $k$, using the same algorithm.

We also note that the exact definition of clustering meaningfulness is not 


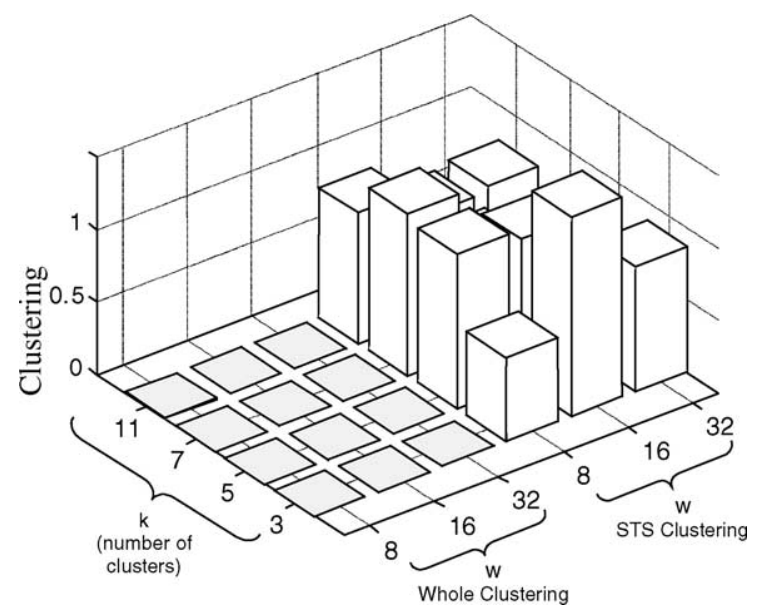

Fig. 2. A Comparison of the Clustering Meaningfulness for Whole Clustering, and STS Clustering, Using $k$-Means With a Variety of Parameters. Note: The two datasets used were Standard and Poor's 500 Index closing values and random walk data.

used. In our definition, each cluster center in $A$ maps onto its closest match in $B$. It is possible, therefore, that two or more cluster centers from $A$ map to one center in $B$, and some clusters in $B$ have no match. However, we tried other variants of this definition, including pairwise matching, minimum matching and maximum matching, together with dozens of other measurements of clustering quality suggested in the literature (Halkidi et al., 2001); it simply makes no significant difference to the results.

\subsection{Hierarchical Clustering}

The previous section suggests that $k$-means clustering of STS time series does not produce meaningful results, at least for stock market data. Two obvious questions to ask are, is this true for STS with other clustering algorithms? And is this true for other types of data? We will answer the former question here and the latter question in Section 3.3.

Hierarchical clustering, unlike $k$-means, is a deterministic algorithm. So we can't reuse the experimental methodology from the previous section exactly, however, we can do something very similar.

First we note that hierarchical clustering can be converted into a partitional clustering, by cutting the first $k$ links (Mantegna, 1999). Figure 3 illustrates the idea. The resultant time series in each of the $k$ subtrees can then be merged into 


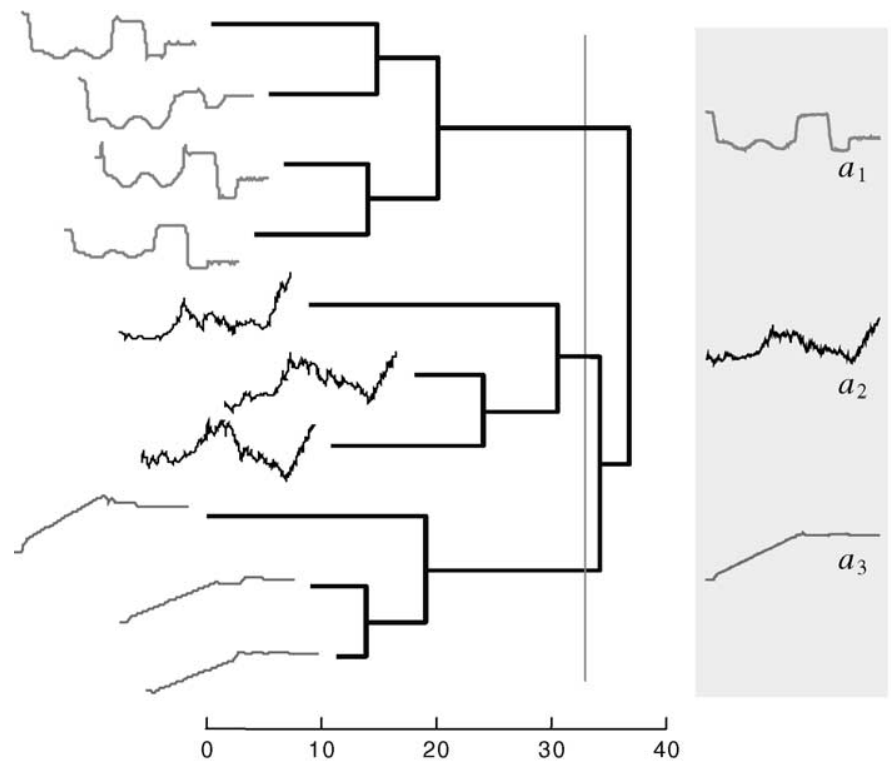

Fig. 3. A Hierarchical Clustering of Ten Time Series. Note: The clustering can be converted to a $k$ partitional clustering by "sliding" a cutting line until it intersects $k$ lines of the dendrograms, then averaging the time series in the $k$ subtrees to form $k$ cluster centers (gray panel).

single cluster prototypes. When performing hierarchical clustering, one has to make a choice about how to define the distance between two clusters; this choice is called the linkage method (cf. step 3 of Table 1).

Three popular choices are complete linkage, average linkage and Ward's method (Halkidi et al., 2001). We can use all three methods for the stock market dataset, and place the resulting cluster centers into set $X$. We can do the same for random walk data and place the resulting cluster centers into set $Y$. Having done this, we can extend the measure of clustering meaningfulness in Eq. (4) to hierarchical clustering, and run a similar experiment as in the last section, but using hierarchical clustering. The results of this experiment are shown in Fig. 4.

Once again, the results are astonishing. While it is well understood that the choice of linkage method can have minor effects on the clustering found, the results above tell us that when doing STS clustering, the choice of linkage method has as much effect as the choice of dataset! Another way of looking at the results is as follows. If we were asked to perform hierarchical clustering on a particular dataset, but we did not have to report which linkage method we used, we could 
reuse an old random walk clustering and no one could tell the difference without re-running the clustering for every possible linkage method.

\subsection{Other Datasets and Algorithms}

The results in the two previous sections are extraordinary, but are they the consequence of some properties of stock market data, or as we claim, a property of the sliding window feature extraction? The latter is the case, which we can simply demonstrate. We visually inspected the UCR archive of time series datasets for the two time series datasets that appear the least alike (Keogh, 2002b). The best two candidates we discovered are shown in Fig. 5.

We repeated the experiment of Section 3.2, using these two datasets in place of the stock market data and the random walk data. The results are shown in Fig. 6.

In our view, this experiment sounds the death knell for clustering of STS time series. If we cannot easily differentiate between the clusters from these two vastly different time series, then how could we possibly find meaningful clusters in any data?

In fact, the experiments shown in this section are just a small subset of the experiments we performed. We tested other clustering algorithms, including EM 


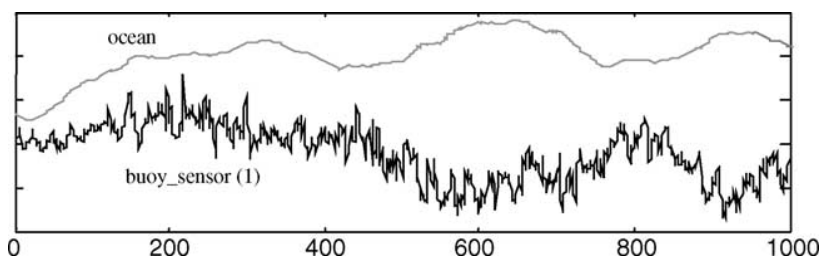

Fig. 5. Two Subjectively Very Dissimilar Time Series from the UCR Archive. Note: Only the first 1000 datapoints are shown. The two time series have very different properties of stationarity, noise, periodicity, symmetry, autocorrelation etc.

and SOMs (van Laerhoven, 2001). We tested on 42 different datasets (Keogh, 2002a; Keogh \& Kasetty, 2002). We experimented with other measures of clustering quality (Halkidi et al., 2001). We tried other variants of $k$-means, including different seeding algorithms. Although Euclidean distance is the most commonly used distance measure for time series data mining, we also tried other distance measures from the literature, including Manhattan, $L_{\infty}$, Mahalanobis distance and dynamic time warping distance (Gavrilov et al., 2000; Keogh, 2002a; Oates, 1999). We tried various normalization techniques, including $Z$ normalization, 0-1 normalization, amplitude only normalization, offset only normalization, no normalization etc. In every case we are forced to the inevitable

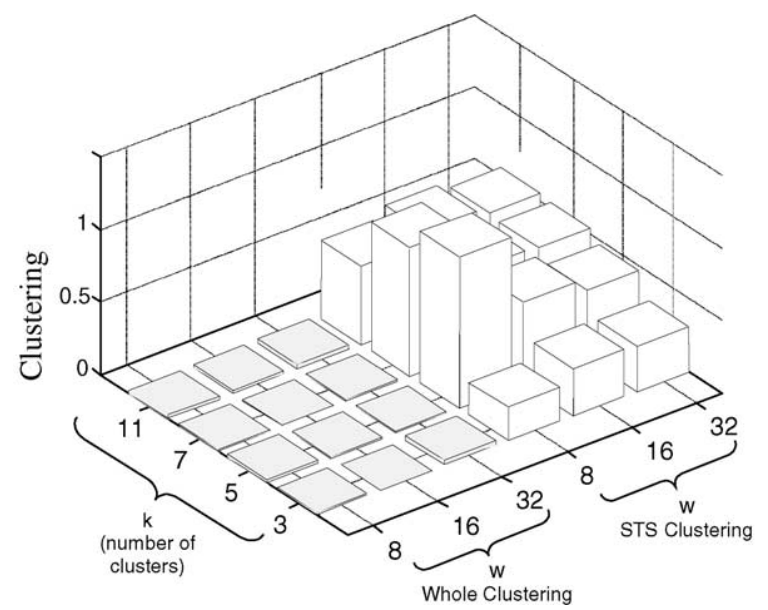

Fig. 6. A Comparison of the Clustering Meaningfulness for Whole Clustering, and STS Clustering, Using $k$-Means With a Variety of Parameters. Note: The two datasets used were buoy_sensor(1) and ocean. 
1 conclusion: whole clustering of time series is usually a meaningful thing to do, but

2 sliding window time series clustering is never meaningful.

\section{WHY IS STS CLUSTERING MEANINGLESS?}

Before explaining why STS clustering is meaningless, it will be instructive to visualize the cluster centers produced by both whole clustering and STS clustering. By definition of $k$-means, each cluster center is simply the average of all the objects within that cluster (cf. step 4 of Table 2). For the case of time series, the cluster center is just another time series whose values are the averages of all time series within that cluster. Apparently, since the objective of $k$-means is to group similar objects in the same cluster, we should expect the cluster center to look somewhat similar to the objects in the cluster. We will demonstrate this on the classic Cylinder-Bell-Funnel data (Keogh \& Kasetty, 2002). This dataset consists of random instantiations of the eponymous patterns, with Gaussian noise added. Note that this dataset has been freely available for a decade, and has been referenced more than 50 times (Keogh \& Kasetty, 2002). While each time series is of length 128 , the onset and duration of the shape is subject to random variability. Figure 7 shows one instance from each of the three patterns.

We generated a dataset that contains 30 instances of each pattern, and performed $k$-means clustering on it, with $k=3$. The resulting cluster centers are shown in
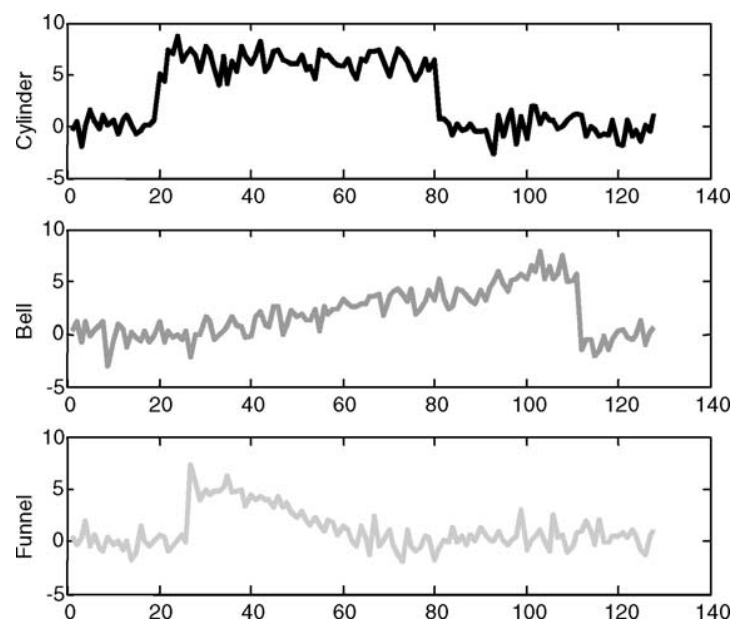

Fig. 7. Examples of Cylinder, Bell, and Funnel Patterns. 


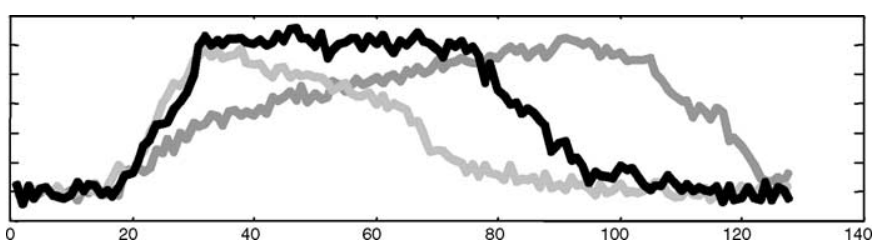

Fig. 8. The Three Final Centers Found by k-Means on the Cylinder-Bell-Funnel Dataset. Note: The shapes of the centers are close approximation of the original patterns.

Fig. 8. As one might expect, all three clusters are successfully found. The final centers closely resemble the three different patterns in the dataset, although the sharp edges of the patterns have been somewhat "softened" by the averaging of many time series with some variability in the time axis.

To compare the results of whole clustering to STS clustering, we took the 90 time series used above and concatenated them into one long time series. We then performed STS clustering with $k$-means. To make it simple for the algorithm, we used the exact length of the patterns $(w=128)$ as the window length, and $k=3$ as the number of desired clusters. The cluster centers are shown in Fig. 9.

The results are extraordinarily unintuitive! The cluster centers look nothing like any of the patterns in the data; what's more, they appear to be perfect sine waves.

In fact, for $w \ll m$, we get approximate sine waves with STS clustering regardless of the clustering algorithm, the number of clusters, or the dataset used! Furthermore, although the sine waves are always exactly out of phase with each other by $1 / k$ period, overall, their joint phase is arbitrary, and will change with every random restart of $k$-means.

This result explains the results from the last section. If sine waves appear as cluster centers for every dataset, then clearly it will be impossible to distinguish one dataset's clusters from another. Although we have now explained the inability

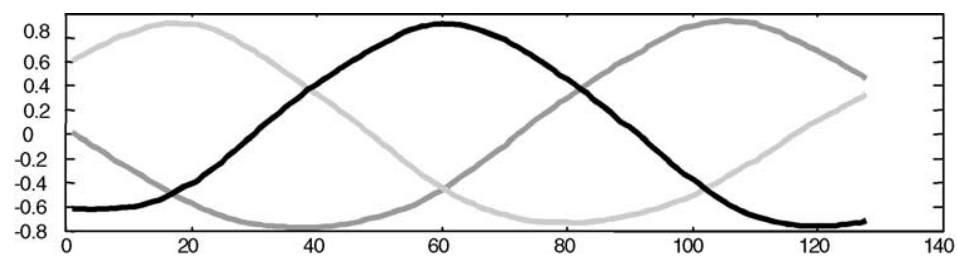

Fig. 9. The Three Final Centers Found by Subsequence Clustering Using the Sliding Window Approach. Note: The cluster centers appear to be sine waves, even though the data itself is not particularly spectral in nature. Note that with each random restart of the clustering algorithm, the phase of the resulting "sine waves" changes in an arbitrary and unpredictable way. 
of STS clustering to produce meaningful results, we have revealed a new question: why do we always get cluster centers with this special structure?

\subsection{A Hidden Constraint}

To explain the unintuitive results above, we must introduce a new fact.

Theorem 1. For any time series dataset $T$ with an overall trend of zero, if $T$ is clustered using sliding windows, and $w \ll m$, then the mean of all the data (i.e. the special case of $k=1$ ), will be an approximately constant vector.

In other words, if we run STS $k$-means on any dataset, with $k=1$ (an unusual case, but perfectly legal), we will always end up with a horizontal line as the cluster center. The proof of this fact is straightforward but long, so we have elucidated it in a separate technical report (Truppel et al., 2003). Note that the requirement that the overall trend be zero can be removed, in which case, the $k=1$ cluster center is still a straight line, but with slope greater than zero. We content ourselves here with giving the intuition behind the proof, and offering a visual "proof" in Fig. 10.

The intuition behind Theorem 1 is as follows. Imagine an arbitrary datapoint $t_{i}$ somewhere in the time series $T$, such that $w \leq i \leq m-w+1$. If the time series is much longer than the window size, then virtually all datapoints are of this type. What contribution does this datapoint make to the overall mean of the STS matrix $S$ ? As the sliding window passes by, the datapoint first appears as the rightmost value in the window, then it goes on to appear exactly once in every possible location within the sliding window. So the $t_{i}$ datapoint contribution to the overall shape is the same everywhere and must be a horizontal line. Only those points at the very beginning and the very end of the time series avoid contributing their value to all $w$ columns of $S$, but these are asymptotically irrelevant. The average of many horizontal lines is clearly just another horizontal line. Another way to look at it is that every value $v_{i}$ in the mean vector, $1 \leq i \leq w$, is computed by averaging essentially every value in the original time series; more precisely, from $t_{i}$ to $t_{m-w+i}$. So for a time series of $m=1,024$ and $w=32$, the first value in the mean vector is the average of $t[1 \ldots 993]$; the second value is the average of $t[2 \ldots 994]$, and so forth. Again, the only datapoints not being included in every computation are the ones at the very beginning and at the very end, and their effects are negligible asymptotically.

The implications of Theorem 1 become clearer when we consider the following well documented fact. For any dataset, the weighted (by cluster membership) average of $k$ clusters must sum up to the global mean. The implication for STS 

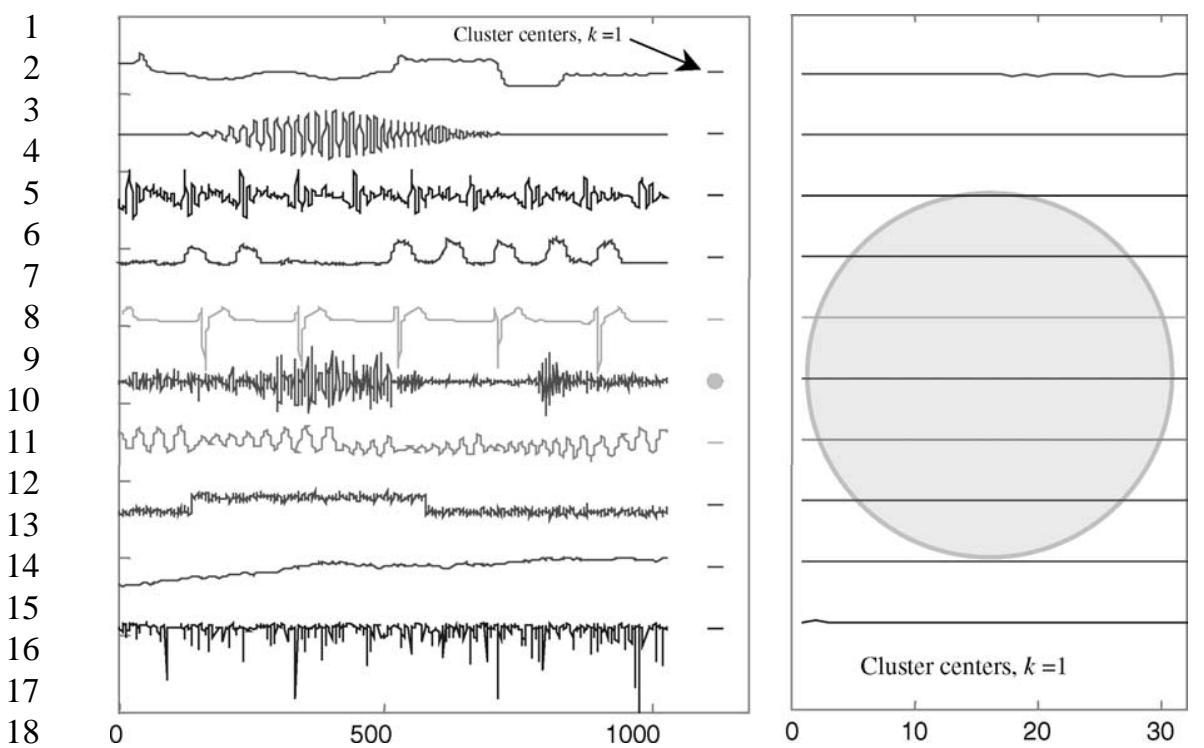

Fig. 10. A Visual "Proof" of Theorem 1. Ten Time Series of Vastly Different Properties of

Stationarity, Noise, Periodicity, Symmetry, Autocorrelation etc. Note: The cluster centers for each time series, for $w=32, k=1$ are shown at right. Far right shows a zoom-in that shows just how close to a straight line the cluster centers are. While the objects have been shifted for clarity, they have not been rescaled in either axis; note the light gray circle in both graphs. The datasets used are, reading from top to bottom: Space Shuttle, Flutter, Speech, Power_Data, Koski_ecg, Earthquake, Chaotic, Cylinder, Random_Walk, and Balloon.

clustering is profound. Since the global mean for STS clustering is a straight line, then the weighted average of $k$-clusters must in turn sum to a straight line. However, there is no reason why we should expect this to be true of any dataset, much less every dataset. This hidden constraint limits the utility of STS clustering to a vanishing small set of subspace of all datasets. The out-of-phase sine waves as cluster centers that we get from the last section conforms to this theorem, since their weighted average, as expected, sums to a straight line.

There are further constraints on the types of datasets where STS clustering could 40

\subsection{The Importance of Trivial Matches} possibly work. Consider a subsequence $C_{p}$ that is a member of a cluster. If we 


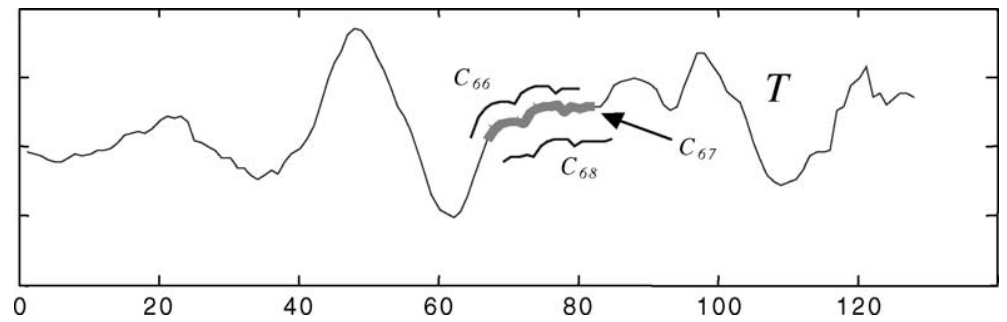

Fig. 11. For Almost Any Subsequence $C$ in a Time Series, the Closest Matching Subsequences are the Subsequences Immediately to the Left and Right of $C$.

examine the entire dataset for similar subsequences, we should typically expect to find the best matches to $C_{p}$ to be the subsequences ..., $C_{p-2}, C_{p-1}, C_{p+1}, C_{p+2}$, ... In other words, the best matches to any subsequence tend to be just slightly shifted versions of the subsequence. Figure 11 illustrates the idea, and Definition 4 states it more formally.

Definition 4 (Trivial Match). Given a subsequence $C$ beginning at position $p$, a matching subsequence $M$ beginning at $q$, and a distance $R$, we say that $M$ is a trivial match to $C$ of order $R$, if either $p=q$ or there does not exist a subsequence $M^{\prime}$ beginning at $q^{\prime}$ such that $D\left(C, M^{\prime}\right)>R$, and either $q<q^{\prime}<p$ or $p<q^{\prime}<q$.

The importance of trivial matches, in a different context, has been documented elsewhere (Lin et al., 2002).

An important observation is the fact that different subsequences can have vastly different numbers of trivial matches. In particular, smooth, slowly changing subsequences tend to have many trivial matches, whereas subsequences with rapidly changing features and/or noise tend to have very few trivial matches. Figure 12 illustrates the idea. The figure shows a time series that subjectively appears to have a cluster of 3 square waves. The bottom plot shows how many trivial matches each subsequence has. Note that the square waves have very few trivial matches, so all three taken together sit in a sparsely populated region of $w$-space. In contrast, consider the relatively smooth Gaussian bump centered at 125. The subsequences in the smooth ascent of this feature have more than 25 trivial matches, and thus sit in a dense region of $w$-space; the same is true for the subsequences in the descent from the peak. So if clustering this dataset with $k$-means, $k=2$, then the two cluster centers will be irresistibly drawn to these two "shapes," simple ascending and descending lines. 

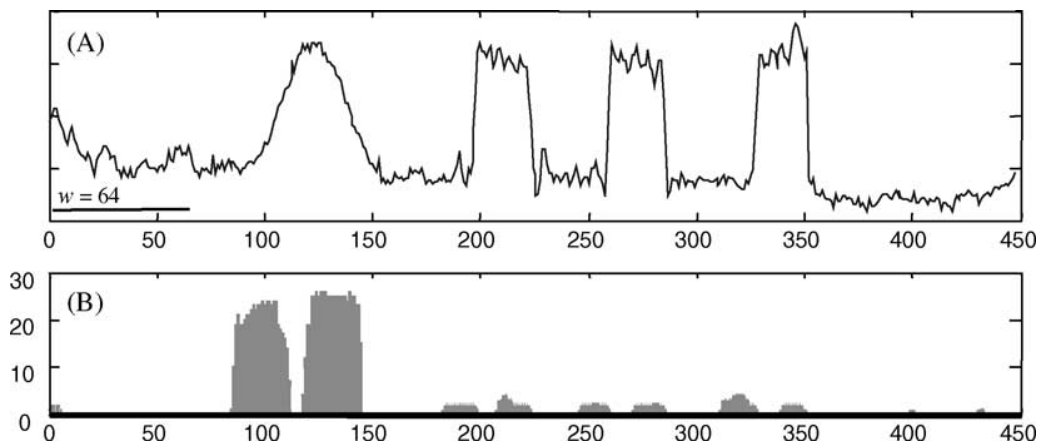

Fig. 12. (A) A Time Series T That Subjectively Appears to Have a Cluster of 3 Noisy Square Waves. (B) Here the $i$ th Value is the Number of Trivial Matches for the Subsequence $C_{i}$ in $T$, where $R=1, w=64$.

The importance of this observation for STS clustering is obvious. Imagine we have a time series where we subjectively see two clusters: equal numbers of a smooth slowing changing pattern, and a noisier pattern with many features. In $w$-dimensional space, the smooth pattern is surrounded by many trivial matches. This dense volume will appear to any clustering algorithm an extremely promising cluster center. In contrast, the highly featured, noisy pattern has very few trivial matches, and thus sits in a relatively sparse space, all but ignored by the clustering algorithm. Note that it is not possible to simply remove or "factor out" the trivial matches since there is no way to know beforehand the true patterns.

We have not yet fully explained why the cluster centers for STS clustering degenerate to sine waves (cf. Fig. 9). However, we have shown that for STS "clustering," algorithms do not really cluster the data. If not clustering, what are the algorithms doing? It is instructive to note that if we perform singular value decomposition on time series, we also get shapes that seem to approximate sine waves (Keogh et al., 2001). This suggests that STS clustering algorithms are simply returning a set of basis functions that can be added together in a weighted combination to approximate the original data.

An even more tantalizing piece of evidence exists. In the 1920s "data miners" were excited to find that by preprocessing their data with repeated smoothing, they could discover trading cycles. Their joy was shattered by a theorem by Evgeny Slutsky (1880-1948), who demonstrated that any noisy time series will converge to a sine wave after repeated applications of moving window smoothing (Kendall, 1976). While STS clustering is not exactly the same as repeated moving window smoothing, it is clearly highly related. For brevity we will defer future discussion of this point to future work. 


\subsection{Is there a Simple Fix?}

Having gained an understanding of the fact that STS clustering is meaningless, and having developed an intuition as to why this is so, it is natural to ask if there is a simple modification to allow it to produce meaningful results. We asked this question, not just among ourselves, but also to dozens of time series clustering researchers with whom we shared our initial results. While we considered all suggestions, we discuss only the two most promising ones here.

The first idea is to increment the sliding window by more than one unit each time. In fact, this idea was suggested by Das et al. (1998), but only as a speed up mechanism. Unfortunately, this idea does not help. If the new step size $s$ is much smaller than $w$, we still get the same empirical results. If $s$ is approximately equal to, or larger than $w$, we are no longer doing subsequence clustering, but whole clustering. This is not useful, since the choice of the offset for the first window would become a critical parameter, and choices that differ by just one timepoint can give arbitrarily different results. As a concrete example, clustering weekly stock market data from "Monday to Sunday" will give completely different cluster patterns and cluster memberships from a "Tuesday to Monday" clustering.

The second idea is to set $k$ to be some number much greater than the true number of clusters we expect to find, then do some post-processing to find the real clusters. Empirically, we could not make this idea work, even on the trivial dataset introduced in the last section. We found that even if $k$ is extremely large, unless it is a significant fraction of $T$, we still get arbitrary sine waves as cluster centers. In addition, we note that the time complexity for $k$-means increases with $k$.

It is our belief that there is no simple solution to the problem of STS-clustering; the definition of the problem is itself intrinsically flawed.

\subsection{Necessary Conditions for STS Clustering to Work}

We conclude this section with a summary of the conditions that must be satisfied for STS clustering to be meaningful.

Assume that a time series contains $k$ approximately or exactly repeated patterns of length $w$. Further assume that we happen to know $k$ and $w$ in advance. A necessary (but not necessarily sufficient) condition for a clustering algorithm to discover the $k$ patterns is that the weighted mean of the patterns must sum to a horizontal line, and each of the $k$ patterns must have approximately equal numbers of trivial matches.

It is obvious that the chances of both these conditions being met is essentially zero. 


\section{A CASE STUDY ON EXISTING WORK}

\section{1. (Not) Finding Rules in Time Series}

The algorithm begins by performing STS clustering. The centers of these clusters are then used as primitives to convert the real-valued time series into symbols, which are in turn fed into a slightly modified version of a classic association rule algorithm (Agrawal et al., 1993). Finally the rules are ranked by their $J$-measure, an entropy based measure of their significance.

The rule finding algorithm found the rules shown in Fig. 13 using 19 months of NASDAQ data. The high values of support, confidence and $J$-measure are offered as evidence of the significance of the rules. The rules are to be interpreted as follows. In Fig. 13(b) we see that "if stock rises then falls greatly, follow a smaller rise, then we can expect to see within 20 time units, a pattern of rapid decrease followed by a leveling out" (Das et al., 1998).

What would happen if we used the TSRF algorithm to try to find rules in random walk data, using exactly the same parameters? Since no such rules should exist by definition, we should get radically different results. ${ }^{1}$ Figure 14 shows one such experiment; the support, confidence and $J$-measure values are essentially the same as in Fig. 13!

This one experiment might have been an extraordinary coincidence; we might have created a random walk time series that happens to have some structure to it. Therefore, for every result shown in the original paper we ran 100 recreations using different random walk datasets, using quantum mechanically generated numbers to insure randomness (Walker, 2001). In every case, the results published cannot be distinguished from our results on random walk data.

The above experiment is troublesome, but perhaps there are simply no rules to be found in stock market. We devised a simple experiment in a dataset that does contain known rules. In particular, we tested the algorithm on a normal healthy electrocardiogram. Here, there is an obvious rule that one heartbeat follows another. 


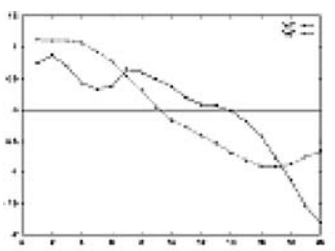

(a)

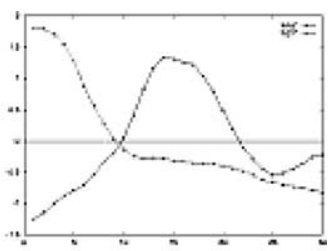

(b)

\begin{tabular}{|c|c|c|c|c|c|c|}
\hline $\mathrm{w}$ & $\mathrm{d}$ & Rule & Sup & Conf & J- & Fig \\
$\%$ & $\%$ & Mea. & \\
\hline 2 & 5. & $7 \Rightarrow^{15} 8$ & 8.3 & 73.0 & 0.00 & (a) \\
0 & 5 & & & & 36 & \\
\hline 3 & 5. & $18 \Rightarrow^{20}$ & 1.3 & 62.7 & 0.00 & (b) \\
0 & 5 & 21 & & & 39 & \\
\hline
\end{tabular}

Fig. 13. Above, Two Examples of "Significant" Rules Found by Das et al. (This is a Capture of Fig. 4 from Their Paper.) Below, a Table of the Parameters They Used and Results They Found.

Surprisingly, even with much tweaking of the parameters, the TSRF algorithm cannot find this simple rule.

The TSRF algorithm is based on the classic rule mining work of Agrawal et al. (1993); the only difference is the STS step. Since the rule mining work has been carefully vindicated in 100s of experiments on both real and synthetic datasets, it seems reasonable to conclude that the STS clustering is at the heart of the problems with the TSRF algorithm.

These results may appear surprising, since they invalidate the claims of a highly referenced paper, and many of the dozens of extensions researchers have proposed (Das et al., 1998; Fu et al., 2001; Harms et al., 2002a, b; Hetland \& Satrom, 2002; Jin et al., 2002a, b; Mori \& Uehara, 2001; Osaki et al., 2000; Sarker et al., 2002; Uehara \& Shimada, 2002; Yairi et al., 2001). However, in retrospect, this result should not really be too surprising. Imagine that a researcher claims to have an algorithm that can differentiate between three types of Iris flowers (Setosa, Virginica and Versicolor) based on petal and sepal length and width ${ }^{2}$ (Fisher, 1936). This claim is not so extraordinary, given that it is well known that even amateur botanists and gardeners have this skill (British Iris Society, 1997). However, the 


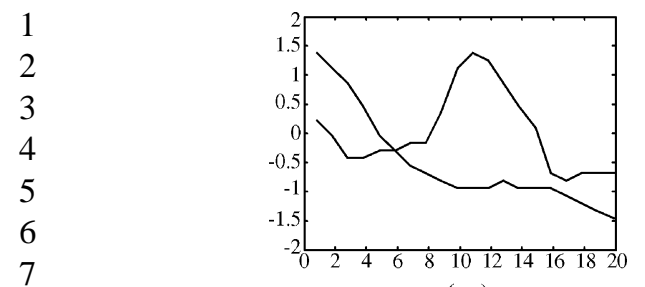
2000; Timmermann et al., 1998). below.

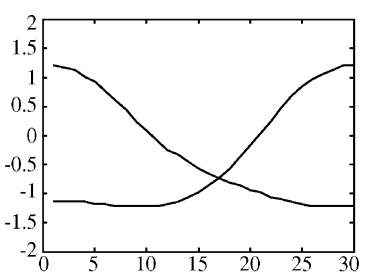

(b)

\begin{tabular}{|c|c|c|c|c|c|c|}
\hline w & d & Rule & $\begin{array}{c}\text { Sup } \\
\%\end{array}$ & $\begin{array}{c}\text { Conf } \\
\%\end{array}$ & $\begin{array}{c}\text { J- } \\
\text { Mea }\end{array}$ & \\
\hline 2 & 5. & $11 \Rightarrow^{15}$ & 6.9 & 71.2 & 0.00 & (a) \\
0 & 5 & 3 & & & 42 & \\
\hline 3 & 5. & $24 \Rightarrow^{20}$ & 2.1 & 74.7 & 0.00 & (b) \\
0 & 5 & 19 & & & 35 & \\
\hline
\end{tabular}

Fig. 14. Above, Two Examples of "Significant" Rules Found in Random Walk Data Using the Techniques of Das et al. Below, We Used Identical Parameters and Found Near Identical Results.

paper in question is claiming to introduce an algorithm that can find rules in stock market time series. There is simply no evidence that any human can do this, in fact, the opposite is true: every indication suggests that the patterns much beloved by technical analysts such as the "calendar effect" are completely spurious (Jensen,

\section{DISCUSSION AND CONCLUSIONS}

As one might expect with such an unintuitive and surprising result, the original version of this paper caused some controversy when first published. Some suggested that the results were due to an implementation bug. Fortunately, many researchers have since independently confirmed our findings; we will note a few

Dr. Loris Nanni noted that she had encountered problems clustering economic times series. After reading an early draft of our paper she wrote "At first we 
1 didn't understand what the problem was, but after reading your paper this fact 2 we experimentally confirmed that (STS) clustering is meaningless!!" (Nanni, 2003). Dr. Richard J. Povinelli and his student Regis DiGiacomo experimentally confirmed that STS clustering produces sine wave clusters, regardless of the dataset used or the setting of any parameters (Povinelli, 2003). Dr. Miho Ohsaki reexamined work she and her group had previously published and confirmed that the results are indeed meaningless in the sense described in this work (Ohsaki et al., 2002). She has subsequently been able to redefine the clustering subroutine in her work to allow more meaningful pattern discovery (Ohsaki et al., 2003). Dr. Frank Höppner noted that he had observed a year earlier than us that “... when using few clusters the resulting prototypes appear very much like dilated and translated trigonometric functions ..." (Hoppner, 2002); however, he did not attach any significance to this. Dr. Eric Perlman wrote to tell us that he had begun to scaling up a project of astronomical time series data mining (Perlman \& Java, 2003); however, he abandoned it after noting that the results were consistent with being meaningless the sense described in this work. Dr. Anne Denton noted, "I've experimented myself, (and) the central message of your paper - that subsequence clustering is meaningless - is very right," and "it's amazing how similar the cluster

\section{CONCLUSIONS}

We have shown that clustering of time series subsequences does not produce meaningful results. We have demonstrated that the choice of clustering algorithms or the measurements of clustering meaningfulness is irrelevant to our claim. We have shown, theoretically and empirically, that the clusters extracted from these time series are forced to obey a certain constraint that is pathologically unlikely to be satisfied by any dataset. More specifically, in order to discover $k$ patterns in any dataset using subsequence clustering, at least two conditions must be satisfied:

(1) the weighted mean of the patterns must sum to a constant line, and

(2) each of the $k$ patterns must have approximately the same number of trivial matches.

Needless to say, the chance of any dataset to exhibit these two properties is very slim.

In future work we intend to consider several related questions; for example, whether or not the weaknesses of STS clustering described here have any implications for model-based, streaming clustering of time series, or streaming clustering of nominal data (Guha et al., 2000). In addition, we plan to investigate 
1 alternatives for finding clusters in time series data. One promising direction is 2 towards time series motif discovery algorithms (Chiu et al., 2003; Lin et al., 2002), 3 which identify frequently occurring patterns in time series.

\section{NOTES}

1. Note that the shapes of the patterns in Figs 13 and 14 are only very approximately sinusoidal. This is because the time series are relatively short compared to the window length. When the experiments are repeated with longer time series, the shapes converge to pure sine waves.

2. This of course is the famous Iris classification problem introduced by R. A. Fischer. It is probably the most referenced dataset in the world.

\section{ACKNOWLEDGMENTS}

We gratefully acknowledge the following people who looked at an early draft of this work. Some of these people were justifiably critical of the work, and their comments lead to extensive rewriting and additional experiments. Their criticisms and comments greatly enhanced the arguments in this paper: Christos Faloutsos, Frank Höppner, Howard Hamilton, Daniel Barbara, Magnus Lie Hetland, Hongyuan Zha, Sergio Focardi, Xiaoming Jin, Shoji Hirano, Shusaku Tsumoto, Loris Nanni, Mark Last, Richard J. Povinelli, Zbigniew Struzik, Jiawei Han, Regis DiGiacomo, Miho Ohsaki, Sean Wang, and the anonymous reviewers of the earlier version of this paper (Keogh et al., 2003). Special thanks to Michalis Vlachos for pointing out the connection between our work and that of Slutsky.

\section{REFERENCES}

Agrawal, R., Imielinski, T., \& Swami, A. (1993, May 26-28). Mining association rules between sets of items in large databases. In: Proceedings of the 1993 ACM SIGMOD International Conference on Management of Data (pp. 207-216). Washington, DC.

Bar-Joseph, Z., Gerber, G., Gifford, D., Jaakkola, T., \& Simon, I. (2002, April 18-21). A new approach to analyzing gene expression time series data. In: Proceedings of the 6th Annual International Conference on Research in Computational Molecular Biology (pp. 39-48). Washington, DC.

Bradley, P. S., \& Fayyad, U. M. (1998, July 24-27). Refining initial points for K-means clustering. In: Proceedings of the 15th International Conference on Machine Learning (pp. 91-99). Madison, WI.

British Iris Society, Species Group Staff (1997). A guide to species irises: Their identification and cultivation. Cambridge University Press. 
Chiu, B., Keogh, E., \& Lonardi, S. (2003, August 24-27). Probabilistic discovery of time series motifs. In: Proceedings of the 9th ACM SIGKDD International Conference on Knowledge Discovery and Data Mining (pp. 493-498). Washington, DC, USA.

Cotofrei, P. (2002, August 24-28). Statistical temporal rules. In: Proceedings of the 15th Conference on Computational Statistics - Short Communications and Posters. Berlin, Germany.

Cotofrei, P., \& Stoffel, K. (2002, April 21-24). Classification rules + time = Temporal rules. In: Proceedings of the 2002 International Conference on Computational Science (pp. 572-581). Amsterdam, Netherlands.

Das, G., Lin, K., Mannila, H., Renganathan, G., \& Smyth, P. (1998, August 27-31). Rule discovery from time series. In: Proceedings of the 4th International Conference on Knowledge Discovery and Data Mining (pp. 16-22). New York, NY.

Denton, A. (2003, December). Personal communication.

Enders, W. (2003). Applied econometric time series (2nd ed.). New York: Wiley.

Fisher, R. A. (1936). The use of multiple measures in taxonomic problems. Annals of Eugenics, 7(2), 179-188.

Fu, T. C., Chung, F. L., Ng, V., \& Luk, R. (2001, August 26-29). Pattern discovery from stock time series using self-organizing maps. In: Workshop Notes of the Workshop on Temporal Data Mining, at the 7th ACM SIGKDD International Conference on Knowledge Discovery and Data Mining (pp. 27-37). San Francisco, CA.

Gavrilov, M., Anguelov, D., Indyk, P., \& Motwani, R. (2000, August 20-23). Mining the stock market: Which measure is best? In: Proceedings of the 6th ACM International Conference on Knowledge Discovery and Data Mining (pp. 487-496). Boston, MA.

Guha, S., Mishra, N., Motwani, R., \& O'Callaghan, L. (2000, November 12-14). Clustering data streams. In: Proceedings of the 41st Annual Symposium on Foundations of Computer Science (pp. 359-366). Redondo Beach, CA.

Halkidi, M., Batistakis, Y., \& Vazirgiannis, M. (2001). On clustering validation techniques. Journal of Intelligent Information Systems (JIIS), 17(2-3), 107-145.

Harms, S. K., Deogun, J., \& Tadesse, T. (2002a, June 27-29). Discovering sequential association rules with constraints and time lags in multiple sequences. In: Proceedings of the 13th International Symposium on Methodologies for Intelligent Systems (pp. 432-441). Lyon, France.

Harms, S. K., Reichenbach, S., Goddard, S. E., Tadesse, T., \& Waltman, W. J. (2002b, May 21-23). Data mining in a geospatial decision support system for drought risk management. In: Proceedings of the 1st National Conference on Digital Government (pp. 9-16). Los Angeles, CA.

Hetland, M. L., \& Satrom, P. (2002). Temporal rules discovery using genetic programming and specialized hardware. In: Proceedings of the 4th International Conference on Recent Advances in Soft Computing (December 12-13). Nottingham, UK.

Honda, R., Wang, S., Kikuchi, T., \& Konishi, O. (2002). Mining of moving objects from time-series images and its application to satellite weather imagery. The Journal of Intelligent Information Systems, 19(1), 79-93.

Hoppner, F. (2002, Sept/Okt). Time series abstraction methods - A survey. In: Tagungsband zur 32. GI Jahrestagung 2002, Workshop on Knowledge Discovery in Databases (pp. 777-786). Dortmund.

Jensen, D. (2000). Data snooping, dredging and fishing: The dark side of data mining. 1999 SIGKDD Panel Report. ACM SIGKDD Explorations, 1(2), 52-54.

Jin, X., Lu, Y., \& Shi, C. (2002a, May 6-8). Distribution discovery: Local analysis of temporal rules. In: Proceedings of the 6th Pacific-Asia Conference on Knowledge Discovery and Data Mining (pp. 469-480). Taipei, Taiwan. 
Jin, X., Wang, L., Lu, Y., \& Shi, C. (2002b, August 12-14). Indexing and mining of the local patterns in sequence database. In: Proceedings of the 3rd International Conference on Intelligent Data Engineering and Automated Learning (pp. 68-73). Manchester, UK.

Kendall, M. (1976). Time-series (2nd ed.). London: Charles Griffin and Company.

Keogh, E. (2002a, August 20-23). Exact indexing of dynamic time warping. In: Proceedings of the 28th International Conference on Very Large Data Bases (pp. 406-417). Hong Kong.

Keogh, E. (2002b). The UCR time series data mining archive. http://www.cs.ucr.edu/ eamonn/ TSDMA/index.html. Computer Science \& Engineering Department, University of California, Riverside, CA.

Keogh, E., Chakrabarti, K., Pazzani, M., \& Mehrotra, S. (2001). Dimensionality reduction for fast similarity search in large time series databases. Journal of Knowledge and Information Systems, 3(3), 263-286.

Keogh, E., \& Kasetty, S. (2002, July 23-26). On the need for time series data mining benchmarks: A Survey and empirical demonstration. In: Proceedings of the 8th ACM SIGKDD International Conference on Knowledge Discovery and Data Mining (pp. 102-111). Edmonton, Alta., Canada.

Keogh, E., Lin, J., \& Truppel, W. (2003, November 19-22). Clustering of time series subsequences is meaningless: Implications for past and future research. In: Proceedings of the 3rd IEEE International Conference on Data Mining (pp. 115-122). Melbourne, FL.

Li, C., Yu, P. S., \& Castelli, V. (1998, November 3-7). MALM: A framework for mining sequence database at multiple abstraction levels. In: Proceedings of the 7th ACM International Conference on Information and Knowledge Management (pp. 267-272). Bethesda, MD.

Lin, J., Keogh, E., Patel, P., \& Lonardi, S. (2002, July 23-26). Finding motifs in time series. In: Workshop Notes of the 2nd Workshop on Temporal Data Mining, at the 8th ACM International Conference on Knowledge Discovery and Data Mining. Edmonton, Alta., Canada.

Mantegna, R. N. (1999). Hierarchical structure in financial markets. European Physical Journal, B11, 193-197.

Mori, T., \& Uehara, K. (2001, September 18-21). Extraction of primitive motion and discovery of association rules from human motion. In: Proceedings of the 10th IEEE International Workshop on Robot and Human Communication (pp. 200-206). Bordeaux-Paris, France.

Nanni, L. (2003, April 22). Personal communication.

Oates, T. (1999, August 15-18). Identifying distinctive subsequences in multivariate time series by clustering. In: Proceedings of the 5th International Conference on Knowledge Discovery and Data Mining (pp. 322-326). San Diego, CA.

Ohsaki, M., Sato, Y., Yokoi, H., \& Yamaguchi, T. (2002, December 9-12). A rule discovery support system for sequential medical data, in the case study of a chronic hepatitis dataset. In: Workshop Notes of the International Workshop on Active Mining, at IEEE International Conference on Data Mining. Maebashi, Japan.

Ohsaki, M., Sato, Y., Yokoi, H., \& Yamaguchi, T. (2003, September 22-26). A rule discovery support system for sequential medical data, in the case study of a chronic hepatitis dataset. In: Workshop Notes of Discovery Challenge Workshop, at the 14th European Conference on Machine Learning/the 7th European Conference on Principles and Practice of Knowledge Discovery in Databases. Cavtat-Dubrovnik, Croatia.

Osaki, R., Shimada, M., \& Uehara, K. (2000). A motion recognition method by using primitive motions. In: H. Arisawa \& T. Catarci (Eds), Advances in Visual Information Management: Visual Database Systems (pp. 117-127). Kluwer. 
Perlman, E., \& Java, A. (2003). Predictive mining of time series data. In: H. E. Payne, R. I. Jedrzejewski \& R. N. Hook (Eds), ASP Conference Series, Vol. 295, Astronomical Data Analysis Software and Systems XII (pp. 431-434). San Francisco.

Povinelli, R. (2003, September 19). Personal communication.

Radhakrishnan, N., Wilson, J. D., \& Loizou, P. C. (2000). An alternative partitioning technique to quantify the regularity of complex time series. International Journal of Bifurcation and Chaos, 10(7), 1773-1779. World Scientific Publishing.

Roddick, J. F., \& Spiliopoulou, M. (2002). A survey of temporal knowledge discovery paradigms and methods. Transactions on Data Engineering, 14(4), 750-767.

Sarker, B. K., Mori, T., \& Uehara, K. (2002). Parallel algorithms for mining association rules in time series data. CS24-2002-1, Tech. Report.

Schittenkopf, C., Tino, P., \& Dorffner, G. (2000, July). The benefit of information reduction for trading strategies. Report Series for Adaptive Information Systems and Management in Economics and Management Society. Report\# 45.

Timmermann, A., Sullivan, R., \& White, H. (1998). The dangers of data-driven inference: The case of calendar effects in stock returns. FMG Discussion Papers dp0304, Financial Markets Group and ESRC.

Tino, P., Schittenkopf, C., \& Dorffner, G. (2000, July). Temporal pattern recognition in noisy nonstationary time series based on quantization into symbolic streams: Lessons learned from financial volatility trading. Report Series for Adaptive Information Systems and Management in Economics and Management Sciences. Report\# 46.

Truppel, W., Keogh, E., \& Lin, J. (2003). A hidden constraint when clustering streaming time series. UCR Tech. Report.

Uehara, K., \& Shimada, M. (2002). Extraction of primitive motion and discovery of association rules from human motion data. In: Progress in Discovery Science, Lecture Notes in Artificial Intelligence (Vol. 2281, pp. 338-348). Springer-Verlag.

van Laerhoven, K. (2001). Combining the Kohonen self-organizing map and K-means for on-line classification of sensor data. In: G. Dorffner, H. Bischof \& K. Hornik (Eds), Artificial Neural Networks, Lecture Notes in Artificial Intelligence (Vol. 2130, pp. 464-470). Springer-Verlag.

Walker, J. (2001). HotBits: Genuine random numbers generated by radioactive decay. http://www. fourmilab.ch/hotbits.

Yairi, Y., Kato, Y., \& Hori, K. (2001, June 18-21). Fault detection by mining association rules in housekeeping data. In: Proceedings of the 6th International Symposium on Artificial Intelligence, Robotics and Automation in Space. Montreal, Canada. 